Corresponding authors: scott.powers@stonybrook.edu; minsig.choi@

stonybrookmedicine.edu

(C) 2020 Rao et al. This article is distributed under the terms of the Creative Commons Attribution-NonCommercial License, which permits reuse and redistribution, except for commercial purposes, provided that the original author and source are credited.

Ontology term: neuroendocrine neoplasm

Published by Cold Spring Harbor Laboratory Press

doi:10.1101/mcs.a004978

\section{Comparative single-cell RNA sequencing (scRNA-seq) reveals liver metastasis-specific targets in a patient with small intestinal neuroendocrine cancer}

\author{
Manisha Rao, ${ }^{1} \mathrm{Ki}$ Oh, ${ }^{2}$ Richard Moffitt, ${ }^{1,3}$ Patricia Thompson, ${ }^{1}$ Jinyu Li, ${ }^{1}$ \\ Jingxuan Liu, ${ }^{1}$ Aaron Sasson, ${ }^{2}$ George Georgakis, ${ }^{2}$ Joseph Kim, ${ }^{2,4}$ Minsig Choi, ${ }^{2}$ \\ and Scott Powers ${ }^{1}$ \\ ${ }^{1}$ Department of Pathology, ${ }^{2}$ Department of Medicine, ${ }^{3}$ Department of Biomedical Informatics, \\ Stony Brook University, Stony Brook, New York 11794, USA
}

Abstract Genomic analysis of a patient's tumor is the cornerstone of precision oncology, but it does not address whether metastases should be treated differently. Here we tested whether comparative single-cell RNA sequencing (scRNA-seq) of a primary small intestinal neuroendocrine tumor to a matched liver metastasis could guide the treatment of a patient's metastatic disease. Following surgery, the patient was put on maintenance treatment with a somatostatin analog. However, the scRNA-seq analysis revealed that the neuroendocrine epithelial cells in the liver metastasis were less differentiated and expressed relatively little SSTR2, the predominant somatostatin receptor. There were also differences in the tumor microenvironments. RNA expression of vascular endothelial growth factors was higher in the primary tumor cells, reflected by an increased number of endothelial cells. Interestingly, vascular expression of the major VEGF receptors was considerably higher in the liver metastasis, indicating that the metastatic vasculature may be primed for expansion and susceptible to treatment with angiogenesis inhibitors. The patient eventually progressed on Sandostatin, and although consideration was given to adding an angiogenesis inhibitor to her regimen, her disease progression involved non-liver metastases that had not been characterized. Although in this specific case comparative scRNA-seq did not alter treatment, its potential to help guide therapy of metastatic disease was clearly demonstrated.

[Supplemental material is available for this article.]

\section{INTRODUCTION}

Metastatic tumor cells were once presumed to be highly similar to primary tumor cells, but there is increasing evidence that primary and metastatic tumor cells from the same patient can be different both genetically and epigenetically (Roe et al. 2017; Yates et al. 2017; Mogensen et al. 2018). Additionally, metastatic tumor microenvironments and their response to therapy can vary substantially between different organ sites (Zhang and Yu 2019). Comparative single-cell RNA sequencing (scRNA-seq) offers a new methodologic

\footnotetext{
${ }^{4}$ Present address: Department of Surgery, University of Kentucky, Lexington, Kentucky 40536, USA
} 
approach to assess differences between the composition of primary and metastatic tumor cells and microenvironments including how signaling between different cell types within the tumor has been altered. These differences might suggest new treatment strategies tailored for metastatic disease, including patients with advanced gastrointestinal neuroendocrine tumors (GI-NETs). GI-NETs are slow-growing tumors arising from neuroendocrine cells located in the GI tract. They are generally well-differentiated with a lack of significant mutations in major driver genes such as TP53, RB1, KRAS, and PTEN (Scarpa et al. 2017; Simbolo et al. 2018). The current standard-of-care treatment is surgical debulking in patients with locally restricted or limited metastases followed by the administration of somatostatin analogs. Somatostatin analogs are used to counteract the effects of neuroendocrine tumor hormone secretion and stabilize disease without major changes in tumor size (Díez et al. 2013). Patients with well-differentiated distant metastases have a 5 -yr survival rate of $35 \%$; if poorly differentiated, the 5 -yr survival rate is $4 \%$, providing motivation to seek new treatments tailored to metastatic tumors (Díez et al. 2013).

\section{RESULTS}

\section{Clinical}

A 58-yr-old female was admitted to Stony Brook Hospital in early 2018 with abdominal pain associated with nausea and vomiting. A computed tomography (CT) scan showed a partial small bowel obstruction. The CT scan also revealed multiple nodules in the liver, small bowel, and peritoneum. In February 2018, the patient had an image-guided liver biopsy performed. Pathology of the liver biopsy revealed a well-differentiated neuroendocrine tumor (carcinoid tumor) with focal necrosis, consistent with metastasis. Post-discharge, the patient briefly tolerated a normal diet and had normal flatulence and regular bowel movements, but she then experienced loose bowel movements and nausea and was then seen in the Surgical Oncology clinic, where she denied having fevers, chills, chest pain, shortness of breath, or vomiting. The case was presented in a multidisciplinary tumor board and the options of hormonal therapy, targeted therapy, locoregional therapy, and surgical debulking were discussed. After counseling with the patient and based on her relatively young age and her symptoms, it was decided that she would benefit from tumor debulking surgery, which was performed in March 2018, during which both the primary small intestine neuroendocrine tumor and the liver metastasis were removed. Since then, the patient has been on monthly injections of $30 \mathrm{mg}$ Sandostatin LAR (octreotide acetate). Recently her cancer progressed and she was put on a daily regimen of $10 \mathrm{mg}$ Afinitor, an mTOR inhibitor, and is currently doing well.

\section{Genomic Analysis}

Separate single-cell RNA libraries using the 10x Genomics platform were prepared from the primary tumor and the liver metastasis. Both tumors were designated as welldifferentiated based on histological analysis. The libraries were sequenced, and following read alignment and generation of single-cell barcode matrices, we performed integrated comparative analysis using Seurat (Stuart et al. 2019). Cluster analysis of the integrated data revealed seven major cell types present in both the primary and metastatic tumors: immune cells, endothelial cells, fibroblasts, and four subtypes of neuroendocrine tumor cells (Fig. 1A). The four neuroendocrine subtypes were designated by Gene Ontology (GO) term enrichment analysis of differentially expressed genes as secretory, synaptic, presynaptic, and stressed or stress response. Based on gene expression analysis, the secretory, presynaptic, and synaptic clusters corresponded to different stages of neuroendocrine differentiation (Fig. 1B). The synaptic subtype differentially expressed nine genes 
A
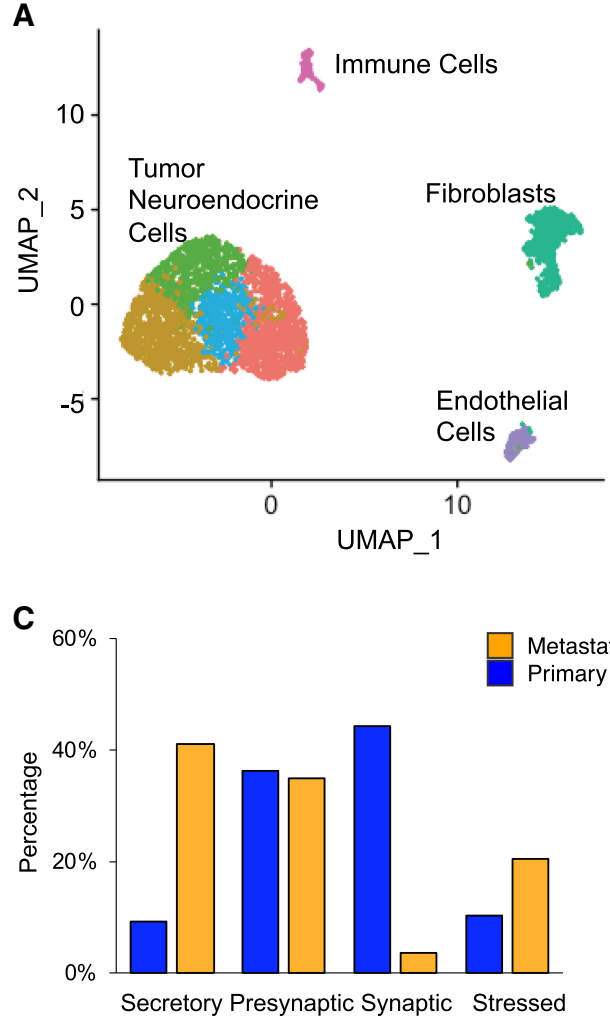

B

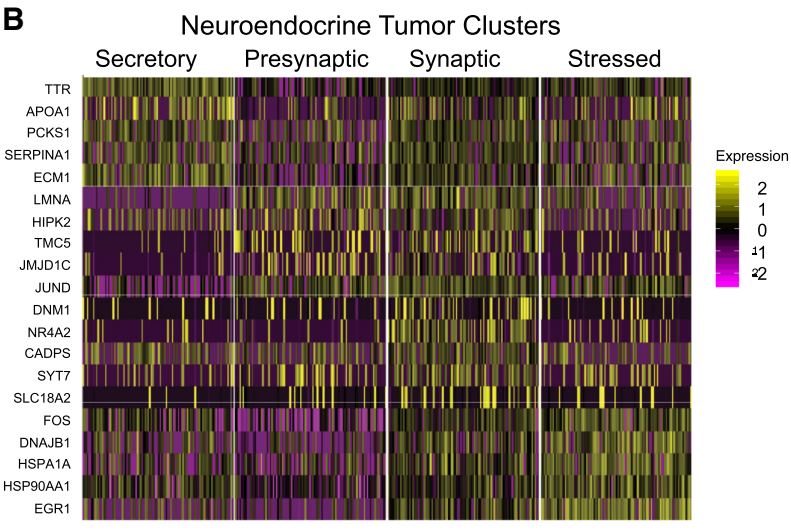

D
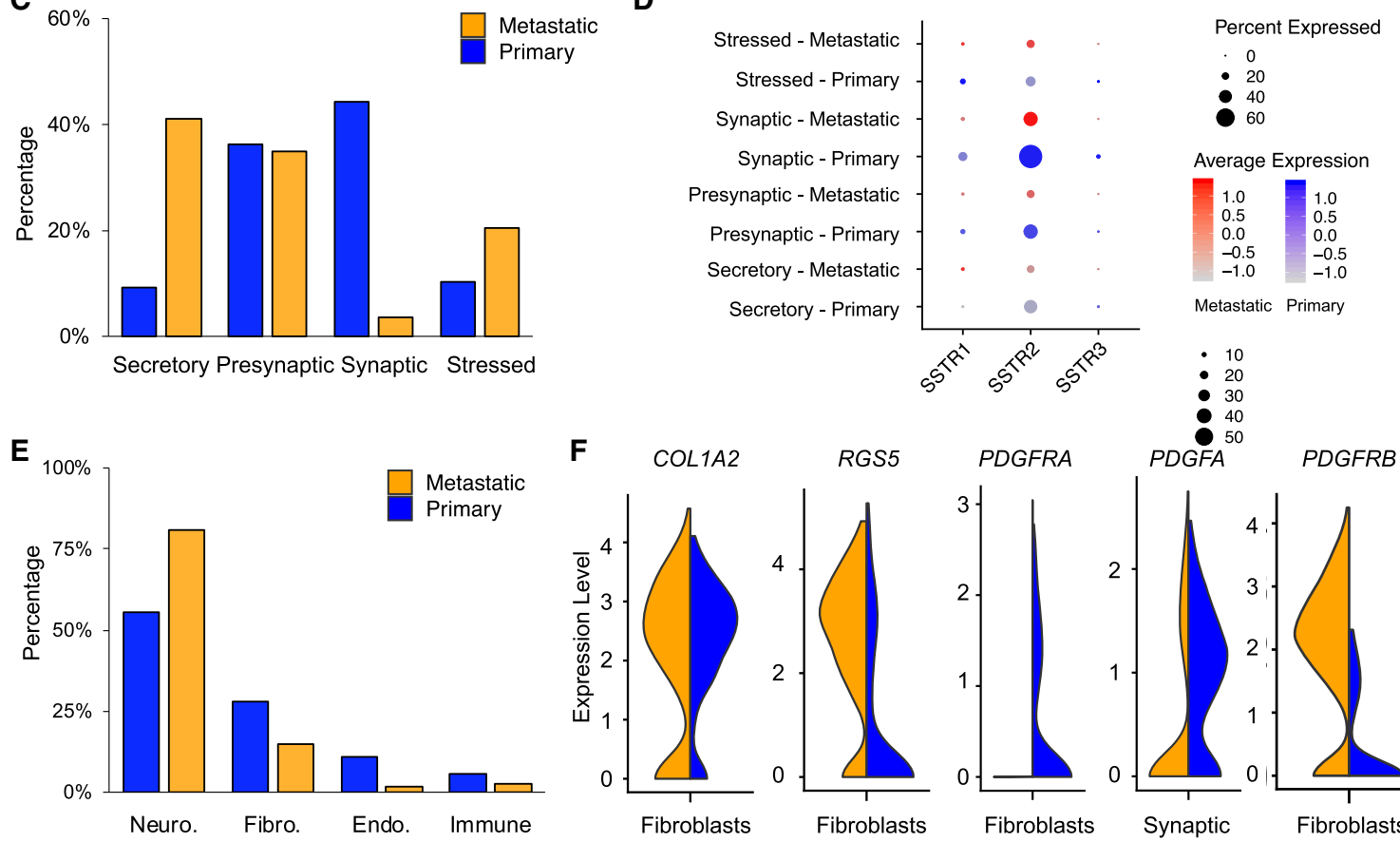

$\mathbf{F}$

COL1A2 RGS5
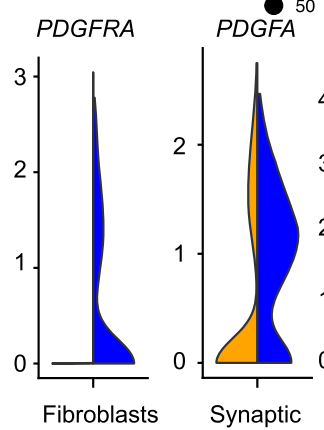

PDGFRB
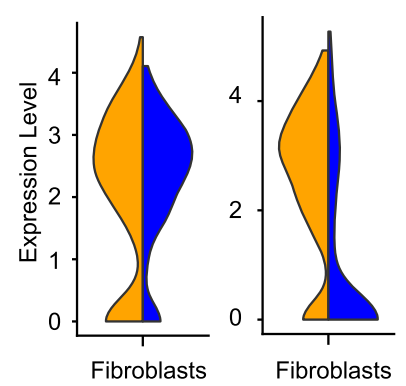

Synaptic

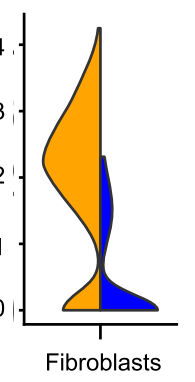

Figure 1. Comparative scRNA-seq analysis of a matched primary and metastatic GI-NET. (A) UMAP projection of the integrated single-cell data showing seven distinct clusters; $(B)$ a heatmap showing the relative expression in the four neuroendocrine subtypes of the top differentially expressed genes; $(C)$ a comparison of the primary and metastatic percentages of the four subtypes of neuroendocrine tumor cells; $(D)$ a dot plot showing comparative primary tumor metastasis expression of somatostatin receptor genes; $(E)$ a comparison of the primary and metastatic percentages of neuroendocrine tumor cells (Neuro.), fibroblast-like cells (Fibro.), endothelial cells (Endo.), and immune cells; and (F) comparative (primary/metastatic) expression of key genes in the indicated tumor cell type.

encoding synaptic proteins or proteins essential for synaptic function, four of which are shown in Figure 1B (DNM1, CADPS, SYT7, and SLC18A2). The presynaptic subtype differentially expressed three synaptic genes, and the secretory subtype expressed one. Also in support of this proposed differentiation hierarchy, $44 \%$ of the neuroendocrine cells of the primary tumor belonged to the most differentiated synaptic subgroup compared to only $2 \%$ of the neuroendocrine cells of the liver metastasis (Fig. 1C). Additionally, $41 \%$ of 
COLD SPRING HARBOR Molecular Case Studies
Comparative scRNA-seq of a patient's metastatic cancer tumor cells of the metastasis belonged to the least differentiated secretory subtype compared to only $9 \%$ of the primary tumor cells (Fig. 1C). Importantly, genes encoding somatostatin receptors, which are the targets of the somatostatin analogs used to slow disease progression, are more strongly expressed in the differentiated synaptic subgroup than they are in the less-differentiated subgroups, resulting in relatively little of this key therapeutic target being expressed in the metastasis (Fig. 1D).

The metastasis had higher tumor cellularity ( $81 \%$ vs. $55 \%)$ and correspondingly fewer stromal cells: less fibroblasts (15\% vs. $28 \%$ ), fewer immune cells (3\% vs. $6 \%$ ), and a sixfold lower percentage of endothelial cells (<2\% vs. 11\%) (Fig. 1E). Within the fibroblast cluster, the expression of COL1A2 appeared similar, but the metastatic tumor fibroblasts expressed considerably more RGS5 (Fig. 1F), a G-protein regulator that is exclusively expressed in pericytes, contractile cells resembling fibroblasts that surround blood vessel endothelial cells (Mitchell et al. 2008). Differential expression analysis of scRNA-seq data has to take into account the cells without a given mRNA ("dropouts"), and the bimodal distributions observed in the plots such as Figure $1 \mathrm{~F}$ reflect the presence of these dropouts and do not reflect a second population of cells with lower expression. We estimated that $80 \%$ of the metastatic "fibroblasts" were actually pericytes, compared to $<25 \%$ pericytes for the primary tumor. Consistent with the greater number of fibroblasts in the primary tumor, the primary tumor showed a possible paracrine inductive interaction between the most differentiated synaptic tumor cells (expressing PDGFA) and fibroblasts (expressing PDGFRA), but the metastasis did not (Fig. 1F). In contrast, the metastatic pericytes expressed considerably higher levels of PDGRB, a known pericyte marker (Fig. 1F). The greater number of fibroblasts in the primary tumor was reflected histologically in the higher amount of collagen-rich stroma observed in the primary tumor (Fig. 2A) compared to the metastasis (Fig. 2B).

We then examined how the primary and metastatic tumor microenvironments differentially impact endothelial cells and vascularization. First, we examined the production of the three VEGF homologs. VEGFA was expressed at higher levels in the neuroendocrine tumor cells and the immune cells of the primary tumor compared to the metastatic tumor (Fig. 2C). VEGFA is known to be expressed by tumor-associated macrophages (TAMs), and the differential expression may indicate a lower influx of TAMs at the metastatic site (Ugel et al. 2015). VEGFB was expressed at high levels in the fibroblasts of the primary tumor but not in its metastatic counterparts and TGFB1, whereas VEGFC was expressed at comparable levels in endothelial cells (Fig. 2D). In contrast, the metastatic endothelial cells expressed higher levels of the VEGF receptor gene FLT1 and significantly higher levels of $K D R$, the latter being the primary VEGF receptor for mitogenic signaling (Fig. 2D). Additionally, the metastatic endothelial cells produced PDGFB, which the primary tumor endothelial cells did not, establishing a possible paracrine signaling support for the metastatic PDGFRB-expressing pericytes (Fig. 2D).

The majority of the protumorigenic genes for secreted proteins we uncovered were expressed at higher levels in the primary tumor but not the metastatic tumor, consistent with the notion that these protumorigenic microenvironmental changes developed in the primary tumor over time (Fig. 2E). These included increased production of VEGFA by both tumor cells and macrophages, increased production of VEGFB by fibroblasts, increased production of PDGFA by tumor cells, and correspondingly higher levels of PDGFRA in fibroblasts. Despite the primary tumor producing more VEGF, the cellular components of the metastatic vasculature appeared primed for expansion (Fig. 2E).

\section{DISCUSSION}

Our study demonstrates that comparative scRNA-seq provides a wealth of information not only on the cell-type composition of metastatic tumors, but also details concerning 
A

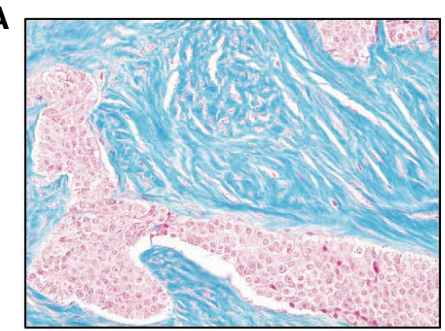

B

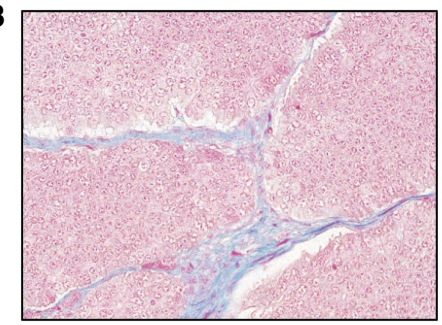

C

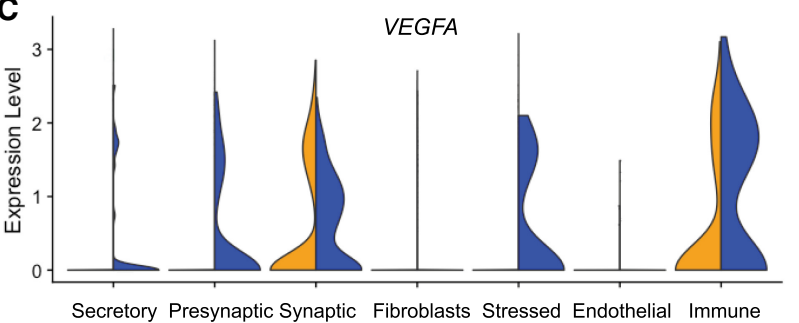

D

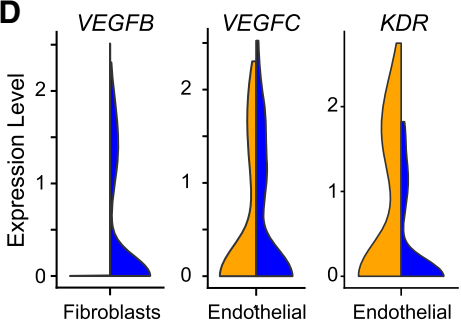

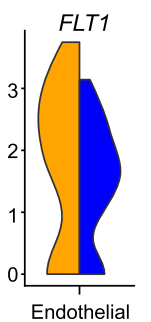

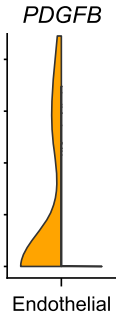

Metastatic
Primary

$\mathbf{E}$

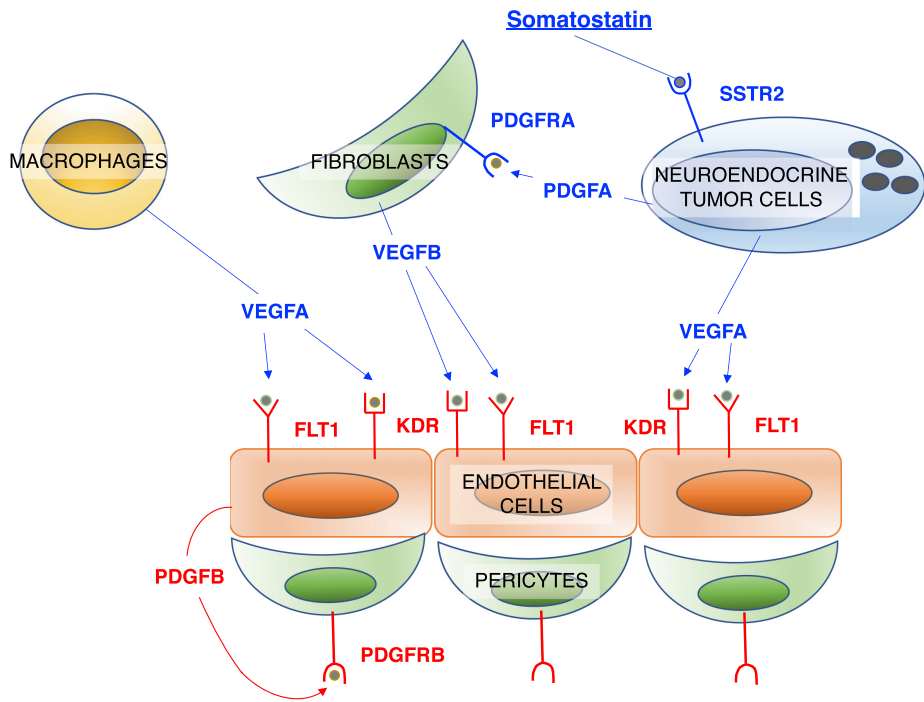

Figure 2. Histological and molecular differences between the primary NET and its metastatic counterpart. (A) Masson's trichrome staining of a primary tumor tissue slice $(40 \times)$; (B) Masson's trichrome staining of a metastatic tumor tissue slice; $(C)$ comparative (primary/metastatic) expression of VEGFA in different tumor cell types; $(D)$ comparative (primary/metastatic) expression of key genes in the indicated tumor cell type; and (E) key differences in gene expression of ligands and corresponding receptors in the tumor microenvironments of primary versus the metastatic gastrointestinal neuroendocrine tumor samples. Blue labeling indicates enhanced expression levels in the primary tumor; red labeling indicates enhanced expression levels in the metastatic tumor.

possible paracrine signaling loops and how this information might be used to tailor therapeutic strategy to blocking metastatic growth. One question that arises is whether bulk RNA-seq would have detected these changes as well. To address this, we computationally combined all the cells for each sample to perform a mock bulk RNA-seq. This analysis indicated that only a minority of alterations would have been detected by bulk RNA-seq (Supplemental Table 1).

The analysis of this patient's liver metastasis provides a rationale for treating this patient's metastatic disease with the multikinase inhibitor sunitinib, which inhibits both PDGFRB, 
COLD SPRING HARBOR Molecular Case Studies
Comparative scRNA-seq of a patient's metastatic cancer expressed preferentially in the metastatic tumor and part of a reciprocal ligand-receptor interaction between endothelial cells and pericytes, and the major mitogenic VEGF receptor encoded by $K D R$, which is also selectively overexpressed in the metastatic endothelium. This interpretation of comparative scRNA-seq results is supported by the preclinical results with sunitinib, which revealed delays in tumor growth in a RIP1-Tag2 transgenic mouse model of pancreatic islet-cell tumors by reducing endothelial-cell density and pericyte coverage of tumor vessels in concert with delays in tumor growth in a mouse model of pancreatic islet-cell tumors (Pietras and Hanahan 2005). However, we do not know whether non-liver metastases would have a similar tumor microenvironment, and treatment with sunitinib may be totally ineffective against those non-liver tumors. Despite sunitinib being approved for pancreatic NET, there is yet no clinical evidence that sunitinib is effective for the treatment of small bowel NET, whereas there is strong evidence that $\mathrm{mTOR}$ inhibition can be effective in halting cancer progression in small bowel NET (Yao et al. 2016).

Despite these caveats, comparative scRNA-seq does appear to have the potential to help advance our understanding of different metastatic microenvironments. Once the cost of comparative scRNA-seq is sufficiently reduced to allow its use in the clinic, its potential application to guiding specific treatments will become clearer.

\section{METHODS}

Tumor Sample Dissociation into a Single-Cell Suspension

The primary tumor specimen and the metastatic lesions were delivered from the surgical pathology core, once the margins were declared negative, in advanced DMEM/F12 on ice. The specimens were washed in ice-cold PBS and minced into $5-\mathrm{mm}^{3}$ sections. For each tissue section, a small section was fixed in $10 \%$ formalin for histological studies. Both the tissue specimens were processed simultaneously to avoid batch effect. The remainder tissue specimens were minced into smaller pieces of $0.5-1 \mathrm{~mm}^{3}$ and digested for $45 \mathrm{~min}$ at $37^{\circ} \mathrm{C}$, in a solution containing $5 \mathrm{mg} / \mathrm{mL}$ Collagenase Type $I I$ (Invitrogen) and $1 \mathrm{mg} / \mathrm{mL}$ dispase (Invitrogen), in the presence of $2.5 \%$ fetal bovine serum (FBS) (Gemini Bioproducts). The tissues were additionally digested with $1 \mathrm{mg} / \mathrm{mL}$ DNAse (Stem Cell Technologies) for $15 \mathrm{~min}$. The digested cells were pelleted and washed two times, and any visible blood cells were removed using the ACK lysis buffer (Sigma-Aldrich). These suspensions were filtered separately through a 70- $\mu \mathrm{m}$ cell strainer to get rid of undigested tissue chunks. If the suspension needed further declumping, it was digested for a minute in TryPLE express (Invitrogen), neutralized with $10 \%$ FBS, and filtered through a $40-\mu \mathrm{m}$ cell strainer. The final cell suspensions were pelleted by centrifuging at $300 \mathrm{~g}$ for $5 \mathrm{~min}$ and resuspended in PBS with $0.1 \%$ BSA (Life Technologies) to a final concentration of $10,00,000$ cells $/ \mathrm{mL}$. The cell viability was examined using trypan blue exclusion (Invitrogen).

\section{scRNA-seq and Sequencing Library Construction Using the 10x Genomics Platform}

Ten thousand cells each from the single-cell suspensions were loaded onto the 10x genomics platform, to generate single-cell encapsulation in gel bead in emulsion (GEM). scRNA-seq libraries were further generated using the Chromium Single Cell 3' Library and Gel Bead kit v2 (PN-120237), as previously described. Libraries were paired-end sequenced on an Illumina HiSeq 4000 instrument.

\section{Bioinformatic Processing of scRNA-seq Data}

The Cell Ranger Single-Cell Suite 3.01 was used to demultiplex and identify the assigned barcodes for each of the primary tumor and metastatic lesions. We used Cell Ranger version 
COLD SPRING HARBOR Molecular Case Studies
Comparative scRNA-seq of a patient's metastatic cancer
1.3.1 (10x Genomics) to process raw sequencing data and Cell Ranger R kit version 2.0.0 and Seurat suite version 3.0.0 for downstream analysis. We obtained reads from 1114 cells from the primary tumor and 3595 cells from the metastatic tumor. For the two samples, total reads between 200 million to 450 million per library was obtained with an average of approximately 40,000 reads and 17,000 reads mapping to an individual cell in each sample, respectively. For more detail on sequencing coverage, see Supplemental Table 2. To test whether the discrepancy in read depth influenced the differential gene expression analysis, both the primary and metastatic counts were equalized using the SampleUMI Seurat tool. The results of this analysis are shown in Supplemental Figure 1. The basic UMAP hyperparameters that we used were n_neighbors $=30$, min_dist $=0.3, n \_$components $=2$, and metric $=$ cosine.

\section{Tissue Preparation of Histological Sectioning, Fixation, and Staining}

The tissue sections preserved for histology purposes fixed in formalin were switched to $70 \%$ ethanol within $24 \mathrm{~h}$ and were sent to the Stony Brook Histology Core for paraffin embedding. Five-micron-thick sections were sectioned and stained with hematoxylin and eosin as well as Masson's trichrome stain according to standardized protocols by the Stony Brook Histology Core. Images were captured at $40 \times$ magnification and reviewed by a pancreatic cancer pathologist.

\section{ADDITIONAL INFORMATION}

\section{Data Deposition and Access}

Single-cell sequencing data generated in the course of this study have been deposited to the Gene Expression Omnibus (GEO) (https://www.ncbi.nlm.nih.gov/geo/) under accession GSE140312.

\section{Ethics Statement}

This study was approved by Stony Brook University Human Subjects committee (IRB), Board Ref\# 2017-4223-F and 2017-4223-R1. The patient provided an informed written consent to perform the comparative transcriptomic analysis.

\section{Acknowledgments}

We thank members of the Powers' laboratory for their help and discussion.

\section{Author Contributions}

M.R. was involved in the planning the project, performed experiments, interpreted data, and revised the manuscript; K.O., R.M., and J. Li performed bioinformatic analysis; A.S., G.G., and J.K. performed surgical oncology; J. Liu provided expert advice in histological analysis; P.T. provided guidance and had a major role in revising the manuscript; J.K., M.C., and S.P. conceptualized the project and had major roles in revising the manuscript; and S.P. supervised the project and wrote the first draft.

\section{Funding}

This work was supported by a grant from the National Institutes of Health (NIH) to S.P. (CA217206) and by the Tissue Analytics and Bioinformatics Shared Resources of the Stony Brook Cancer Center.

Received November 13, 2019; accepted in revised form February 3, 2020. 


\section{REFERENCES}

Díez M, Teulé A, Salazar R. 2013. Gastroenteropancreatic neuroendocrine tumors: diagnosis and treatment. Ann Gastroenterol 26: 29-36.

Mitchell TS, Bradley J, Robinson GS, Shima DT, Ng YS. 2008. RGS5 expression is a quantitative measure of pericyte coverage of blood vessels. Angiogenesis 11: 141-151. doi:10.1007/s10456-007-9085-x

Mogensen MB, Rossing M, Østrup O, Larsen PN, Heiberg Engel PJ, Jørgensen LN, Hogdall EV, Eriksen J, Ibsen $P$, Jess $P$, et al. 2018. Genomic alterations accompanying tumour evolution in colorectal cancer: tracking the differences between primary tumours and synchronous liver metastases by whole-exome sequencing. BMC Cancer 18: 752. doi:10.1186/s12885-018-4639-4

Pietras K, Hanahan D. 2005. A multitargeted, metronomic, and maximum-tolerated dose "chemo-switch" regimen is antiangiogenic, producing objective responses and survival benefit in a mouse model of cancer. $J$ Clin Oncol 23: 939-952. doi:10.1200/JCO.2005.07.093

Roe JS, Hwang Cl, Somerville TDD, Milazzo JP, Lee EJ, Da Silva B, Maiorino L, Tiriac H, Young CM, Miyabayashi K, et al. 2017. Enhancer reprogramming promotes pancreatic cancer metastasis. Cell 170: 875-888 e820. doi:10.1016/j.cell.2017.07.007

Scarpa A, Chang DK, Nones K, Corbo V, Patch AM, Bailey P, Lawlor RT, Johns AL, Miller DK, Mafficini A, et al. 2017. Whole-genome landscape of pancreatic neuroendocrine tumours. Nature 543: 65-71. doi:10.1038/ nature21063

Simbolo M, Vicentini C, Mafficini A, Fassan M, Pedron S, Corbo V, Mastracci L, Rusev B, Pedrazzani C, Landoni $L$, et al. 2018. Mutational and copy number asset of primary sporadic neuroendocrine tumors of the small intestine. Virchows Arch 473: 709-717. doi:10.1007/s00428-018-2450-x

Stuart T, Butler A, Hoffman P, Hafemeister C, Papalexi E, Mauck WM III, Hao Y, Stoeckius M, Smibert P, Satija R. 2019. Comprehensive integration of single-cell data. Cell 177: 1888-1902. doi:10.1016/j.cell.2019.05.031

Ugel S, De Sanctis F, Mandruzzato S, Bronte V. 2015. Tumor-induced myeloid deviation: when myeloid-derived suppressor cells meet tumor-associated macrophages. J Clin Invest 125: 3365-3376. doi:10.1172/ $\mathrm{JCl} 80006$

Yao JC, Fazio N, Singh S, Buzzoni R, Carnaghi C, Wolin E, Tomasek J, Raderer M, Lahner H, Voi M, et al. 2016. Everolimus for the treatment of advanced, non-functional neuroendocrine tumours of the lung or gastrointestinal tract (RADIANT-4): a randomised, placebo-controlled, phase 3 study. Lancet 387: 968-977. doi:10.1016/S0140-6736(15)00817-X

Yates LR, Knappskog S, Wedge D, Farmery JHR, Gonzalez S, Martincorena I, Alexandrov LB, Van Loo P, Haugland HK, Lilleng PK, et al. 2017. Genomic evolution of breast cancer metastasis and relapse. Cancer Cell 32: 169-184 e167. doi:10.1016/j.ccell.2017.07.005

Zhang C, Yu D. 2019. Suppressing immunotherapy by organ-specific tumor microenvironments: what is in the brain? Cell Biosci 9: 82. doi:10.1186/s13578-019-0349-0 


\section{COLD SPRING HARBOR Molecular Case Studies}

\section{Comparative single-cell RNA sequencing (scRNA-seq) reveals liver metastasis-specific targets in a patient with small intestinal neuroendocrine cancer}

Manisha Rao, Ki Oh, Richard Moffitt, et al.

Cold Spring Harb Mol Case Stud 2020, 6: a004978 originally published online February 13, 2020

Access the most recent version at doi: $10.1101 / \mathrm{mcs} .0004978$

$\underset{\text { Material }}{\text { Supplementary }} \quad \begin{gathered}\text { http://molecularcasestudies.cshlp.org/content/suppl/2020/02/18/mcs.a004978.D } \\ \text { C1 }\end{gathered}$

References This article cites 12 articles, 1 of which can be accessed free at:

http://molecularcasestudies.cshlp.org/content/6/2/a004978.full.html\#ref-list-1

License This article is distributed under the terms of the Creative Commons Attribution-NonCommercial License, which permits reuse and redistribution, except for commercial purposes, provided that the original author and source are credited.

Email Alerting Receive free email alerts when new articles cite this article - sign up in the box at the Service top right corner of the article or click here. 